\title{
The delicate balance: mass customisation for large-scale organisational change
}

\section{William Seidman* and Michael McCauley}

Cerebyte, Inc.,

P.O. Box 1674, Lake Oswego, OR 97935, USA

Fax: (503)-624-1642

E-mail: William.Seidman@Cerebyte.com

E-mail: Michael.McCauley@Cerebyte.com

*Corresponding author

\begin{abstract}
Headquarters and local work units almost always approach change initiatives from different perspectives. This can create substantial challenges for organisation leaders, managers and workers. Principles of mass customisation, along with the latest science, can now be applied to these change initiatives, providing a direct and effective way to maintain the balance between headquarters and local requirements. Recent research and technology advances provide a scientific approach to organisational transformations based on mass customisation. Using this approach, almost any organisation can achieve rapid, repeatable transformational results.
\end{abstract}

Keywords: mass customisation; organisational change; positive deviance; neuroscience; fair process; leadership; persuasive technology; organisational transformation; motivation; wisdom; transformational leadership; transactional leadership; grassroots change.

Reference to this paper should be made as follows: Seidman, W. and McCauley, M. (2011) 'The delicate balance: mass customisation for large-scale organisational change', Int. J. Mass Customisation, Vol. 4, Nos. 1/2, pp.67-80.

Biographical notes: William Seidman is a recognised leader and expert on how to develop and sustain high performing organisations. In particular, he is renowned for understanding the processes required to discover and use expert wisdom to create extraordinary organisational performance. $\mathrm{He}$ obtained his Doctorate degree from Stanford where he studied management decision-making. As part of his doctoral dissertation, he developed a groundbreaking technique for analysing management decision-making. The technique has been recognised by KMWorld, The Innovation Center, IDC and others. His credentials include more than 20 years experience as a manager in high technology companies including HP, Silicon Graphics, and Mentor Graphics. As the CEO of Cerebyte, he led engagements for Intel, Jack in the Box, and many others. He is an experienced Consultant and Manager of fast growth, high technology environments.

Michael McCauley is a recognised expert on organisational persuasive technology, web-based application development and social media usage. Prior to co-founding Cerebyte, he spent more than 20 years leading numerous high performance teams at leading-edge companies such as Compaq Computer, Dell Computer, Motorola, and many others. His credits include the development of the first systematic method for rating the maturity of project management processes within organisations which became the foundation for an extensive study of project management maturity conducted by the Project Management 
Institute and U.C. Berkeley. Building on his background as Engineer and Management Consultant, he has developed persuasive technologies that make systematic and sustainable organisational change possible. His undergraduate work was in engineering, he holds an MBA, and is certified by The Project Management Institute as a Project Management Professional.

\section{Introduction}

Major organisational change initiatives commonly generate at least two differing perspectives:

Perspective \#1 You don't understand my (market, office, country, situation, etc.)!

Perspective \#2 We need to have standards, consistency and high quality. We need to deploy our programme efficiently.

Sound familiar? The first perspective is typical of local operating units while the second is typical of headquarters. They are the usual response to most change initiatives.

These differing responses demonstrate both one of the greatest challenges for any strategic change initiative and where mass customisation can make the difference between success and failure for large scale organisational change (defined as impacting at least 200 people). The local perspective almost always focuses on the need to understand and adapt to local requirements and conditions - i.e., a customisation requirement. The headquarters perspective almost always focuses on the need to create consistency and economies of scale - i.e., a mass change requirement. In any major performance improvement initiative, there is a delicate balance between the need for centrally-driven change and the need for individualised solutions adapted to unique local requirements.

Applying the principles of mass customisation to performance improvement and change initiatives is a direct and effective way to find and maintain this delicate balance. Mass customisation is the science of creating a solution that is based on a consistent standard while, at the same time, customising that solution for each individual situation (Pine, 1993) all of which is done at the low cost typical of mass production.

This article presents a methodology that was developed through 15 years of using the principles of mass customisation for strategic performance improvement and change initiatives. More specifically, this article derives primarily from the authors' direct experience guiding large organisations through significant changes and uses recent research and technology in several related fields - positive deviance, fair process, neuroscience and persuasive technology - to provide a scientific approach to organisational transformations based on mass customisation. As such, it is not a formal research paper but a description of paradigms that have emerged from extensive practical experience using mass customisation in a very specific way.

The authors have used this approach in a wide variety of organisations such as the leading manufacturing of semi-conductors, fast food restaurants, healthcare, state and federal agencies and many other types of organisations. It has also been used to drive transformations in organisations that span multiple countries such as the USA, China, Chile, and the UK and in diverse languages, including English, Japanese, Mandarin, and 
Spanish. The success of this approach in these diverse situations is due, to a great degree, to the use of mass customisation techniques.

\section{The need for organisational transformation}

'Change is the only constant'. This is a common statement from executives. An increasingly volatile and demanding world has made organisations' ability to quickly and effectively define and execute new strategies a fundamental criterion for success (or survival). As one executive from a digital advertising company put it: "We will either get really good at changing ourselves in ways that significantly improve performance or we will die".

The pressure to be good at adapting to changing conditions has recently become particularly acute. During the last few years, driven by recession fears and organisational conservatism, most organisations focused a good part of their change initiatives on cutting costs. In this risk-averse environment, relatively few companies consistently pushed forward on significant, positive change initiatives or developed the leadership required to drive such improvements.

Now, however, as organisations emerge from this period, they are discovering that the world is even more complex than before. New technologies, broad regulatory changes and the expanding presence of China and India in global markets are making the executive cliché about change a harsh reality. Organisations must become good at change or they are not likely to survive. Recognising this need for great change leadership, some companies have engaged the authors to assist them in 'developing transformational leadership as a core competency (their words)'.

Yet, organisational change is a very difficult process. Countless books and articles have been written about organisational change including many on how to lead change (cf. Pink, 2009; Heath and Heath, 2010; Rock, 2006; Seidman and McCauley, 2009). Even with all the available information and approaches, there just does not seem to be a 'silver bullet' that quickly and effectively creates organisational change.

One of the reasons change is challenging appears to be the difficulties leadership teams face in finding the proper balance between central control and local autonomy. Too much central control creates a rigid, ineffective bureaucracy that responds poorly to local cultures and regulations. Too much local autonomy creates chaos and undermines an organisation's ability to standardise procedures across geographic areas. Applying mass customisation to organisational transformation efforts gives organisations the ability to quickly and effectively find the optimum balance between these conflicting forces.

\section{Strategic initiatives}

Many major change initiatives are usually in support of a new strategic direction. For example, a fast food restaurant had been competing on food quality and pricing, but realised that its true advantage over the competition was in service. In support of this new strategic focus on improving service, it had to change the way restaurants were managed and customers were served. This restaurant chain launched a strategic initiative designed to improve guest service. Similarly, a company that sold yellow pages advertising 
recognised that yellow pages were a dying market and that they needed to diversify into providing integrated digital and print advertising solutions. This new strategic direction required a change to the entire sales process for 600 sales people. Performance improvement initiatives are commonly used to support specific organisational strategies.

Because such initiatives impact the entire organisation and are often disruptive of the status quo, they are almost always proactively and consciously driven by leaders located in a headquarters functions. Typically, a strategic goal will be identified by executive leadership and a central-organisation-based team will be established to achieve the goal. This team will then develop and execute (or at least try to execute) a company-wide change plan. This is a classic top-down organisational change initiative, which is often used to ensure that the company stays focused and aligned on a limited set of overarching goals.

\subsection{The headquarters perspective}

Although top-down headquarters driven change initiatives have received only minimal attention in recent literature, we encounter many companies that use centralised change strategies. There are good reasons for programmes to be top-down, headquarters driven (Kerzner, 1984). By approaching change centrally, organisations can:

- be unified around a single theme or message to ensure everyone's alignment

- present consistent content to the learner to ensure that everyone learns the same new business attitudes and processes

- deploy the performance improvement programme consistently in order to ensure a predictable result

- be very economical, touching many people at a very low cost per person

- make it easier to transfer personnel across geographic areas

- plan and implement common policies across all divisions and territories.

In short, headquarters-driven programmes seem to have many of the benefits usually associated with mass production. They can produce consistent high quality results at a lower cost per unit. Obviously, this is a very desirable result. Since the organisation needs to improve performance in a selected area, headquarters wants to ensure that everyone understands and executes the new programme efficiently and effectively.

How does headquarters typically develop and deploy such change programmes? The most common approaches to mass transformations use standard project management and mass media techniques. Typically, a small team is identified and chartered to manage the change. They then obtain resources to do the actual work such as defining new business processes, technology or skills and developing training vehicles. For example, the authors worked with a large, global pharmaceutical company that wanted to significantly improve the performance of its supply chain. The company identified a small team of 15 people to plan and execute the programme. Many of these 15 people then led specialised work groups that solicited input from the organisation, defined the new capabilities, developed materials or technology and established a deployment strategy. Even though these teams tried to include local participation, the distances, time zones and travel expenses made the programme look and feel as though it came from headquarters. In spite of efforts to align 
with local needs, many headquarters-driven programmes may be viewed as 'corporate', and may be resisted because of that perception.

In addition, organisations tend to use a limited, well-accepted, but marginally effective set of methodologies to deploy the new processes and/or technologies. The methodologies commonly used to deploy a change programme include:

- executive roadshows

- videos or online presentations

- highly scripted training (ideally delivered locally to keep costs down)

- e-learning solutions.

Each of these has serious drawbacks. Executive roadshows and videos can certainly motivate people in the short-term, but the effect wears away quickly and the learner is left to figure out how to apply the content to their own situation. This reduces their transformative impact. Similarly, scripted training and e-learning can have a good short-term impact, but they are generally more expensive to develop and deliver and have minimal support for application to 'real' situations. Like roadshows and videos, these processes may have little impact on a firm's long-term transformation. Overall, in the authors' experience, these approaches have shown little sustained success at creating meaningful change.

Not surprisingly, the combination of headquarters-intensive content and marginally effective deployment approaches has created a legacy of resistance to headquarters-driven performance improvement initiatives. In many organisations, 'solutions' provided by headquarters are often dismissed out-of-hand and therefore are never truly given a fair chance at success. As a result, the authors often hear headquarters change initiatives described as:

- 'more pontificating from headquarters'

- 'solutions from the ivory tower'

- ' fad of the week'.

It is no surprise then that many mass produced change initiatives are seen as excessively bureaucratic. When presented with one of these initiatives, many employees take an approach of 'ignore it and hope it will go away'.

However, one additional comment about initiatives that is often heard gives a clue to how to overcome these barriers. Frequently, people reject these centrally-driven change initiatives with statements such as: 'You just don't understand...' and fill in the blank with something local like - my market, my customers, my office or my country. This suggests that if the initiative appears to be better aligned with local conditions and requirements, people might be more likely to perceive direct benefit from the initiative and it might be more quickly accepted. In other words, meaningful localisation is a key to change initiative success.

\subsection{The local perspective}

Do you know the expression 'What's in it for me (aka WIIFM)?' Most of us have heard some variation of this perspective when deploying a change initiative. We, as humans, 
seem to be biologically programmed to be more acutely tuned to our personal circumstances and immediate environment than to wider, more abstract concepts (Quinn, 2004). When a change is proposed, no matter how compelling the vision or the need, the first response is often self-interest. What will I get out of it? Will it help me or hurt me? Will it work in my situation or will it cause me grief?

In organisations, WIIFMs often surface as an emphasis on local differences. For example, a logistics operation that moves products throughout Asia and Europe has to contend with unique customs clearance processes in virtually every country. Similarly, but a bit more subtly, a global hotel chain was driving an initiative that was meant to ensure that every customer was appropriately greeted upon check in. Initially, an 'appropriate' greeting was defined as a US style enthusiastic, high-energy greeting, which was considered to be rude by some cultures. As the chain responded to this feedback, it introduced a quieter, Japanese style greeting, complete with bow, which some US guests saw as remote. The nature of the greeting had to be uniquely suited to the culture where the hotel is located and even, if possible, to the culture of the client checking in. Overarching headquarters initiatives require significant, often very subtle adjustments, to meet local requirements during implementation.

In fact, there is hardly an issue where local variation is not important to functioning. Features and benefits, pricing, introductions, service expectations, gifts, all vary not just in different countries but even within a company operating in just one country. The differences in language, attitudes, and business processes between the engineering, manufacturing, marketing, sales and services groups can be staggering. Even though an organisation has a strong corporate culture, each group sees the world through a very well-developed and unique lens. These individual 'visions' are naturally wary of mass change programmes, especially when they are not specifically adapted to their own perspective. Given these individual perspectives, it is reasonable to place intense focus on localisation.

In our experience, few companies have systems specifically designed to promote local adaptation. Instead local entities within the company simply make the adjustments they feel are appropriate, in many cases ignoring headquarters direction. After all, the real action in an organisation is at the local level where headquarters has little meaningful impact. Particularly, when a local group is in a different part of the world, facing a different market, the need to adapt is real and often beyond headquarters' ability to proactively manage.

Local adaptation is not, however, a panacea for change initiatives. When there is too much local adaptation, chaos may rein. In one photography studio company with six divisions, for example, the local differences in the divisions had become so extreme that each division used a different camera, production processes, facilities and business processes and none of these could be interchanged or easily linked. As a result, at some times of the year, the school sports division would be completely overloaded, while the holidays and religious events divisions were almost idle. Due to different equipment and training, workers in the slow divisions could not be shifted to the division in need of additional personnel and equipment. Conversely, at other times the reverse was true - these same divisions were swamped while others were under-utilised. Efforts to change these divisions to better align with each other were met with: 'You don't understand my market'. Consequently, change initiatives were almost completely rejected. Overall, allowing each division to completely localise the change initiative seriously hurt the company. 
Similarly, when a large semi-conductor manufacturer introduced a new, global sales and pricing programme, one region immediately and without significant analysis changed it to fit their perceptions of the local markets. This included departing from the global standard for the sales process and pricing for multi-national clients. Suddenly, the manufacturing company was getting uncomfortable questions from its multi-national customers about why some groups within their organisation were getting a particular programme and pricing while other groups were getting something quite different and more costly for the same product and service. Ultimately, the manufacturing company was forced to match the lowest global price for everyone, at considerable reduction to overall profitability. Our experience with many organisations suggests that adapting virtually any change initiative to fit a perception of local requires is an instantaneous but sometimes incorrect response.

\subsection{The delicate balance}

The conundrum for leadership of organisational change is now apparent. As Figure 1 illustrates, developing and maintaining a balance between centralisation and local autonomy optimises the impact of initiatives. Conversely, moving too far toward either end of the change continuum tends to undermine the success of any change initiative. Too much centralisation (the left side of the graph) can produce bureaucracy and reduces local responsiveness to real world variation leading to a suboptimal change effort. Too much local adaptation (the right side of the graph) produces little transference across divisions, inconsistent quality standards and denies a firm the benefits of economies of scale, again leading to a suboptimal performance improvement. Although there may be slight variation around the central balancing point, any significant movement away from the centre tends to generate an adverse impact.

Figure 1 A continuum for mass change

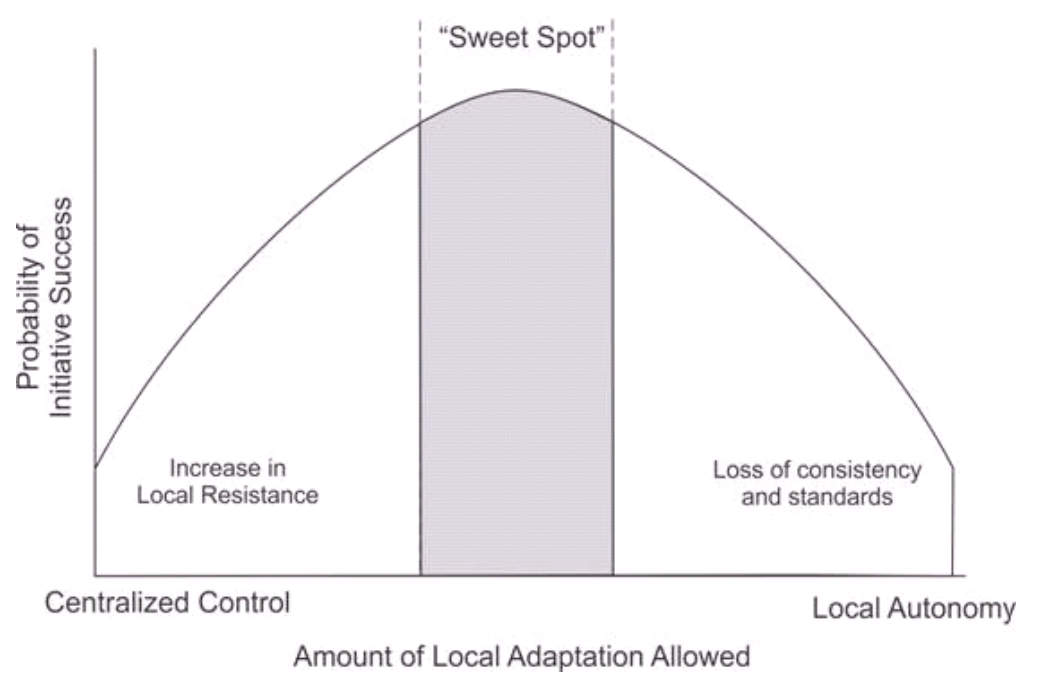

Where is the 'sweet spot' that optimally balances these conflicting forces? How can an organisation quickly and effectively find that sweet spot for any change initiative? 
These are questions that mass customisation can answer. In an optimal situation, change initiatives need to be mass produced to capture all of the consistency, quality standards and economies of scale found in headquarters-driven changes while at the same time enabling each individual and local entity to appropriately adapt it to unique local requirements.

\section{A model for mass transformation}

By integrating the principles of mass customisation with recent developments in three research domains, plus the emergence of new 'persuasive' technology designed on the principles of mass customisation, it is now possible for organisations to quickly and effectively find their mass transformation sweet spot. More specifically, the science has led the authors to develop a four-part model of organisational change (Figure 2) that leverages the principles of mass customisation.

Figure 2 Four part model of change

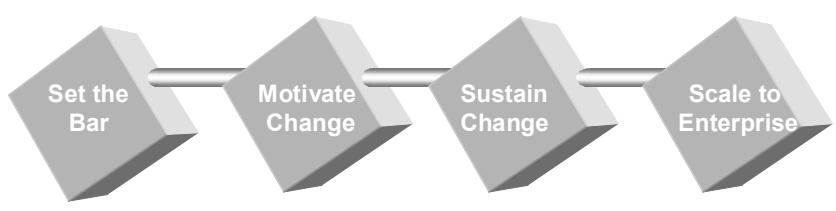

The first component, set-the-bar, is the foundation for the 'mass' part of the mass transformation. The second component, motivation, focuses on individual customisation to create engagement. The third part, sustaining, concentrates on adapting and customising the programme to specific local circumstances. The fourth part, scaling, describes how the first three boxes can be done for changes that effect many people who may be spread around the world.

\subsection{Set-the-bar}

Any transformation begins with a clear image of what extraordinary performance should be as a result of the change. The best way to build this image is to leverage the 'wisdom' of the 'positive deviants' in the organisation. Positive deviants are the select few people in an organisation who consistently and systematically out perform all the others. As such, they are the primary source of what is often described as the 'tribal wisdom' of the organisation (Pascale et al., 2010; Pascale and Sternin, 2006). We will focus on those elements of positive deviant knowledge that are the drivers of both the mass elements in a transformation and the foundation for the customisation.

Extensive work with leveraging positive deviants as the foundation for mass transformation suggests that there are four critical areas of their attitudes, thought patterns and behavioural patterns that are important for driving change. These are their:

- focus on achieving a 'greater social good' as their primary objective of the work transformation

- $\quad$ organisation of the transformation effort into a series of major developmental phases or 'big steps' that help structure the process 
- detailing of the key concepts that specify the desired outcome of each big step in a set of 'principles' that guide a variety of potential applications of the new knowledge

- specifying a series of highly practical, applied learning tasks (including potential supporting resources) that are the optimum process for the organisation learning the new attitudes, thoughts and behaviours.

Mapping these elements of positive deviant knowledge to the four-part change model, the first three elements provide a conceptual framework that will be used primarily for individual customisation in the motivating step. In motivating, each individual will adapt the positive deviant concepts to integrate with their own belief system. The last element provides the foundation for more situational customisation which occurs in the sustaining part of the model. In sustaining, individuals and teams adapt the positive deviant knowledge to their specific situation.

\subsection{Mass motivation}

Mass motivation is a particular challenge in large-scale transformations. An organisation must motivate each individual, regardless of their personal background or interest to embrace a transformation. If the motivational efforts are too heavy handed, the initiative is rejected as an inappropriate imposition. If the motivation is insufficient, no one engages and the change flounders.

Fortunately, the principles of mass customisation can guide the application of fair process (Kim and Mauborgne, 2003) and the neuroscience of positive visualisation (Assaraf and Smith, 2008; Rock and Schwartz, 2006) to the positive deviant wisdom in a way that produces mass motivation. More specifically, by having each participant review the positive deviant social good statement, big steps and principles and re-write these in their own words, people come to see themselves in the same context as the positive deviants. The transposition of the positive deviant content (the mass portion) into personal language engages people and the writing drives integration of the positive deviant content with the personal content (the customisation portion). For example, recall the fast food restaurant chain that was deploying a new customer service initiative to its 1,400 restaurants. We observed that, at the level of the objective, big steps and principles, all 1,400 restaurant managers embraced the conceptual framework (again, the mass portion), but found it useful to personalise the language to fit their unique situation, experience and learning style (again, the customisation portion). Not surprisingly, after modifying the positive deviant objective, big steps and principles into their own language, each restaurant manager thought these were their own ideas, but the content was actually mass produced. Because of mass customisation, the change looked and felt like a grassroots driven effort, but was actually driven by corporate.

\subsection{Mass sustaining}

The initial embracing of a change using mass customisation does not produce a sustained transformation. While the initial mass motivation is a significant achievement, the principles of mass customisation need to be used to drive the changes required for each unique environment. More specifically, the principles of mass customisation are now 
used to guide the application of additional areas of neuroscience for mass sustained impact.

Mass customisation of the repetitive, practice learning tasks and utilising the resources identified by the positive deviants gives us the means of achieving transformation on a large scale. Here is how it works. A team of people from the same group or office who are participating in the transformation examine the recommended learning tasks. They are directed to:

- Determine the learning and/or application intent of each learning task by answering the question 'What is this trying to teach me?'.

- Once they determine the intent, they answer the question: 'How can this be adapted to my work environment?' The expectation is to achieve the intent but in a way that is very appropriate for them.

- Finally, they commit to a date for completing their now personalised learning and/or application task by answering the question: 'How long will it take me to complete each task?'.

Whose learning task is it now? Clearly, it is theirs (the customisation portion) but it is based on achieving the intent from the positive deviant wisdom (the mass portion). The dates can then be centrally tracked (another mass element) to ensure that each individual (remember they set the dates) is executing a plan that will achieve the complete transformation. As each individual and team goes to actually perform the learning tasks, they are individually changing, which culminates in a mass transformation.

Continuing the fast food restaurant example, while all 1,400 restaurant managers had an essentially identical conceptual framework (objective, big steps and principles), their development plans and schedules were uniquely tailored to each restaurant. The intent was achieved on a mass basis, but the implementation was completely customised.

\subsection{Scaling}

While it may be possible to do the above processes without technology in small, co-located environments, it is virtually impossible to do it on a large-scale and in global environments. Technology is essential for making mass transformations successful.

Recent technology breakthroughs are providing capabilities that were not possible even just a few years ago. In particular, the development of 'persuasive technologies', most of which are designed around the principles of mass customisation, is revolutionising the ability to drive large-scale transformations. Persuasive technologies are designed to influence what people believe and do (Fogg, 2003). As such, they use the mass capability to touch many people, while enabling each individual to interact with them uniquely.

As applied to this methodology, persuasive technology is used to guide interactions with the positive deviant content. The positive deviant wisdom is stored in a library and presented to the learner with a series of cues that guide the learner into interacting with the content and documenting their personalisation online. The system also sends prompts to the learner about their learning tasks helping them to keep on schedule. The persuasive technology provides a structure that drives consistent predictable mass customisation and, as a result, efficient organisational change. 


\section{Mass customisation for transformations}

What does this look like when is it working correctly? Here are a few examples.

The authors worked with an auto parts chain with more than 3,000 stores developing and implementing a programme to improve store management's ability to grow its consumer business. The chain had substantial evidence that by restructuring the store management team from a store manager with one general assistant to a store manager with both a parts assistant and a consumer assistant, there would be a significant increase in both sales and employee satisfaction. However, making this change was challenging because most of the people in the stores loved working on their own cars and therefore preferred working in the parts department. Historically too, almost all of the senior management of the company had come up through the parts department, so parts was generally perceived as a better growth path for employees. Not surprisingly, there was widespread resistance to the recommended change.

But some positive deviant store managers had made the transition with minimal resistance and good sales results. Following the processes outlined here, the positive deviants established a compelling objective statement, clear, powerful big steps and strong principles defining each major step. They also listed a solid set of learning experiences to guide other store managers in making the change. Again using this approach, the positive deviant best practices were deployed and uniquely tailored to each store.

The results illustrated the value of using mass customisation for organisational change. At the level of the concepts contained in the positive deviants' objective, big steps and principles, all the participating stores were effectively identical. They were all working from and toward the same conceptual framework. However, at the level of the specific implementation plans, each was completely unique to the specific store. While the concepts were mass produced, the deployment in each store was completely customised.

This situation was part of a tightly controlled proof case with test and comparison stores. More specifically, 16 stores using this approach where matched by sales demographics and experience of the management team with 16 stores in the same region (to control for the effects of the regional manager) and 16 stores in another region. The stores were compared on two measures: sales against budget and inventory loss. The stores following this approach showed a 5.25\% increase in sales in 12 weeks (the defined test period) and demonstrated 30\% improvement in 'lost' inventory over the comparison stores. Benchmarks from other retail environments indicated that these numbers were quite significant, a bottom-line result that executives in the company attributed to using mass customisation.

This approach also works for international situations, where both language and culture can be issues. The authors worked with a global manufacturing company that was deploying a new customer service initiative to 450 service personnel in five centres around the world that was aimed at improving working relationships with clients. Historically, the relationship between the company's customers and the service organisation was mostly transactional. The customer would contact the service group and place an order, which would be processed by the service group. However, with increasingly complex products and decreasing lead-times, this transactional approach was no longer working. Orders were changing too frequently and the customer service group 
was getting overwhelmed. The company decided that shifting the relationship between the customer and the service group to a more collaborative model would allow a better exchange of information resulting in more stable and effective order management processes.

A group of positive deviant customer service people from around the world assembled in the UK to develop new business processes for the evolving business. They identified the need for a new attitude and model of the business for customer service as well as streamlined operational procedures. Their objective, big steps and principles were clear and powerful. The foundation for the 'mass' part was established.

However, the deployments had to adapt to significant local variations. While the USA, Japan and the People's Republic of China (PRC) functioned as a single team, both European (EMEA) and Asia-Pacific (APAC) service operations were each comprised of small teams servicing specific countries or regions. For example, EMEA operations included teams for the major countries - UK, France, and Germany - as well as teams that focused on 'Eastern Europe', 'the Middle East' or 'Africa'. Similarly, in APAC, there were teams dedicated to Korea and Taiwan, while other teams handled broad regions such as 'Southeast Asia' that included Malaysia, Vietnam, Singapore and other countries in the region. The differences between the large country-focused teams and the regionally focused teams were huge and challenging. 'One size' was definitely not going to 'fit all' in this environment.

Customisation occurred on multiple levels. The original positive deviant best practices were first adapted by local positive deviants to each major region so there was a tailored set of best practices for the USA, EMEA, PRC, APAC and Japan. Surprisingly, there were very few real differences in the objective, big steps and principles at this level. A few words were changed to better fit local language (e.g., different words were used to describe collaboration in Japan), but all of the concepts stayed the same. Again, the mass part was evident.

However, the task lists and application of the new processes were considerably different, though always within the intent of the learning activities. For example, while all of the groups included elements of collaboration, the way it was implemented in the USA and for the group of countries included in 'Eastern Europe' was quite different. Even the specific exercises were unique. Similarly, because APAC, had some teams located in Hong Kong while others were in their local countries, each team had to contemplate the intent of the programme independently and adapt it uniquely to their own situation. The programme was systematically customised to accommodate a wide variety of different conditions and circumstances, all without losing the intent.

As a result of mass customisation, headquarters achieved a consistent economical world-wide programme, while each local entity obtained a programme uniquely adapted to their situations. In more formal terms, the programme was evaluated over a six month period by comparing teams that completed the programme with teams that did not use the programme on the accuracy of the team's 'tactical demand forecast'. Teams using this approach were twice as good on key metrics as comparison groups using more traditional approaches. Mass customisation had a real impact. 


\section{Conclusions}

Few would dispute the increasing globalisation of our world. Many companies have thousands of people operating in hundreds of locations. Even small and medium-sized companies operating within markets may have multiple sales offices, service centres and production facilities. Whenever an organisation has both a headquarters and other operating units, there is the opportunity for conflict between central and local interests and forces and, of course, an opportunity to apply mass customisation to change initiatives. This has implications for both research and organisational leadership.

While there appear to have been many studies of mass customisation in manufacturing setting, the authors were unable to find any studies that specifically focused on the use of mass customisation for change initiatives in general and, more specifically for change initiatives involving a large groups in diverse locations. With increasing globalisation, understanding the cultural implications of mass customisation might be a fruitful area for research. Similarly, determining if there are size limitations or if the 'sweet spot' of balance varies according to particular factors might increase understanding of the value and use of mass customisation in these areas. Overall, very little is known about local-headquarters conflicts making this a major area of potential of study with huge implications, particularly for global businesses.

Practically, almost any executive will say that effectively leading large-scale change is one of their most challenging issues. These executives struggle constantly to find an effective balance between their need to drive an organisation in a particular direction and the contrary pressures to allow local autonomy and responsiveness. Some executives err on the side of too much central direction. Others give too much autonomy. Few seem to recognise the importance of the 'sweet spot' where there is an optimum balance between local and central control for effectively leading their organisations. Fewer still seem to realise that mass customisation, which is widely discussed and used in manufacturing, could help them lead their organisations to higher performance. The authors' have demonstrated in hundreds of diverse settings that the principles of mass customisation provide organisations with a new and powerful means of driving large scale change and performance improvement initiatives. Mass customisation lets change leaders have the best of both mass and customisation, with strong results to prove it.

\section{References}

Assaraf, J. and Smith, M. (2008) The Answer, Atria Books, New York.

Fogg, B.J. (2003) Persuasive Technology, Morgan Kaufman, San Francisco.

Heath, C. and Heath, D. (2010) SWITCH, Broadwaym, New York.

Kerzner, H. (1984) Project Management: A Systems Approach to Planning, Scheduling, and Controlling, Van Nostrand Reinhold Company, New York.

Kim, W.C. and Mauborgne, R. (2003) 'Fair process: managing in the knowledge economy', Harvard Business Review, January, Vol. 81.

Pascale, R.T. and Sternin, J. (2006) 'Your company's secret change agents', Harvard Business Review, Reprint R0505D. 
Pascale, R.T., Sternin, J. and Sternin, M. (2010) The Power of Positive Deviance, Harvard Business Press, Boston.

Pine, J. (1993) Mass Customization, Harvard Business School Press, Boston.

Pink, D. (2009) DRIVE, Peguin Group, New York.

Quinn, R.E. (2004) 'Building the bridge as you walk on it: a guide for leading change', Jossey Bass, San Francisco.

Rock, D. (2006) Quiet Leadership, Collins, New York.

Rock, D. and Schwartz, J. (2006) The Neuroscience of Leadership, Summer, Strategy + Business.

Seidman, W. and McCauley, M. (2009) Strategy to Action in 10 Days, Advantage Media Group, Charleston. 